\title{
Tracce di una storia della pedagogia del neonato in Europa dall'Umanesimo al primo Novecento
}

Traços de uma história da pedagogia de recém-nascidos na Europa, do Humanismo ao início do século XX

Traces of a history of pedagogy of the newborn in Europe from Humanism to the early twentieth century

Huellas de la historia de la pedagogía del neonato en Europa desde el humanismo hasta principios del siglo $\mathrm{XX}$

\author{
Evelina Scaglia
}

Università degli Studi di Bergamo (Itália)

https://orcid.org/0000-0002-4365-0252

evelina.scaglia@unibg.it

\section{Astratto}

La proposta di scoprire alcune tracce di una storia della pedagogia del neonato in Europa dall'Umanesimo al primo Novecento è finalizzata a mostrare, attraverso la rilettura di alcuni autori significativi, il legame esistente nello sviluppo della loro riflessione pedagogica fra la genesi storica e la manifestazione morfologica dei processi educativi nell'esperienza di ciascuna persona. L'articolo intende affrontare un primo approfondimento di tale questione, nell'ottica di fornire anche un contributo alla formazione dei genitori e degli educatori impegnati nei servizi dell'Early Childhood Education and Care (ECEC).

Parole chiave: Educazione neonatale. Genitorialità. Storia della pedagogia. 


\title{
Resumo
}

A proposta de descobrir alguns vestígios de uma história da pedagogia de recém-nascidos na Europa, do Humanismo ao início do século XX, tem o objetivo de mostrar, por meio da releitura de alguns autores significativos, o vínculo existente no desenvolvimento da sua reflexão pedagógica entre a gênese histórica e a manifestação morfológica dos processos educacionais na experiência de cada pessoa. $\mathrm{O}$ artigo pretende abordar um primeiro estudo sobre esta temática, com o objetivo de contribuir também para a formação de pais e educadores envolvidos em serviços de Educação e Atenção à Primeira Infância (EPAI).

Palavra-chave: Educação neonatal. Paternidade. História da pedagogia.

\begin{abstract}
The purpose of exploring some traces of a history of pedagogy of the newborn in Europe from Humanism to the early twentieth century is to show, through the re-reading of some significant authors, the link that exists in the development of their pedagogical reflections between the historical genesis and the morphological manifestation of the educational processes in the experience of each person. The article intends to address an initial study of this issue, with a view also to providing a contribution to the training of parents and educators involved in Early Childhood Education and Care (ECEC) services.
\end{abstract}

Keywords: Neonatal education. Parenting. History of pedagogy.

\section{Resumen}

La propuesta de descubrir algunas huellas de la historia de la pedagogía del neonato en Europa desde el humanismo hasta principios del siglo XX tiene como objetivo mostrar, a través de la relectura de algunos autores significativos, el vínculo existente en el desarrollo de su reflexión pedagógica entre la génesis histórica y la manifestación morfológica de los procesos educativos en la experiencia de cada persona. El artículo pretende abordar un primer estudio de este tema, a fin de aportar igualmente una contribución a la formación de los padres y educadores implicados en los servicios de Educación y Atención de la Primera Infancia (AEPI).

Palabras clave: Educación neonatal. Parentalidad. Historia de la pedagogía. 


\section{Introduzione}

Proporre uno studio storico-pedagogico dedicato alla progressiva maturazione di una pedagogia dell'età neonatale significa affrontare la sfida di tesaurizzare i contributi offerti da una pluralità di fonti attorno al tema dell'educazione e della formazione dei più piccoli. Se con il sorgere della paideia occidentale, tradizionalmente identificata con l'opera di Omero, la centralità dell'educazione e della formazione dei nuovi nati sul piano morale e tecnico venne riconosciuta in quanto «emanazione diretta della viva coscienza normativa d'una comunità umana» (JAEGER, 2003, p. 25), occorre attendere quantomeno lo sviluppo dell'Umanesimo e l'avvio dell'Età moderna per reperire fonti letterarie, filosofiche, teologiche, artistiche, dalle quali emerga il passaggio dall'adozione di un agire educativo più attento ai piccini alla formulazione di un sapere pedagogico pronto a riconoscerli come portatori di lógos, caratteristica che contraddistingue l'essere umano da qualsiasi altro animale superiore.

Se la storia dell'infanzia, come sottolineato da Lloyd DeMause nell'ambito della sua teoria psicogenetica della storia, si rivelò essere nel corso dei secoli un «incubo» (DEMAUSE, 1974), e come sostenuto da Franco Cambi e Simonetta Ulivieri va fatta rientrare fra $i$ «silenzi nell'educazione» per il processo di marginalizzazione subito da piccoli e piccolissimi (CAMBI; ULIVIERI, 1994), risulta di particolare interesse cogliere la sfida storiografica di varcare le soglie degli spazi domestici che fecero, per lungo tempo, da sfondo alla nascita e alla prima infanzia, per ispezionare un «luogo elettivo» connotato da dimensioni affettive, emotive e sentimentali (COVATO, 2004, p. 53-71). In questo modo, dovrebbe essere più facile identificare le ragioni dell'elaborazione, fra Età moderna e contemporanea, di un pensiero pedagogico capace di rendere conto della costruzione dell'identità infantile attraverso le esperienze di relazione educativa vissute nei primi giorni e mesi di vita con gli adulti di riferimento, e di illustrane le peculiarità rispetto ai meri processi di cura, allevamento, disciplinamento, modellamento o coltivazione.

\section{Lo sguardo nuovo dell'Umanesimo}

L'Età umanistica costituì il primo terreno di sviluppo di una riflessione espressamente pedagogica sull'età neonatale, perché vide i prodromi dello sviluppo di un «sentimento dell'infanzia» (ARIÈS, 1961). In particolare, nel tardo Medioevo d'oltralpe e nel Quattrocento italiano i bambini si muovevano e vivevano quotidianamente all'interno di «spazi della vita adulta». La mescolanza di grandi e piccoli favorì il sorgere di uno sguardo nuovo su ogni piccino che, se anche condivideva nella quotidianità dei ceti meno abbienti le fatiche e le durezze della vita degli adulti, era in ogni caso «oggetto di ascolto, destinatario di gesti affettuosi, attrezzato con un outillage peculiare, di abbigliamento, di gioco, di aiuto alla sua crescita, di cui nelle epoche precedenti non sembra dotato» (BECCHI; JULIA, 1996a, p. 120). Questa specificità era dovuta alla forte importanza riconosciuta alla famiglia, concepita come spazio della domesticità ed ambiente naturale di nascita e crescita del fanciullo regolato dai genitori, ma aperto in un'ideale linea di continuità con l'ambiente extradomestico (DEMAUSE, 1974, p. 186-238). Nell'Italia centro-settentrionale ebbero un particolare peso sia il passaggio storico dalla realtà socio-politica dei comuni alla nascita delle prime Signorie, sia l'entrata sulla scena sociale di nuovi protagonisti come i mercanti (a lungo malvisti dalla cultura di matrice ecclesiastico-nobiliare) e gli artisti (scultori, pittori, cesellatori, ecc.), che incarnavano nella loro vita attiva l'idealtipo dell'homo faber fortunae suae e fecero da apripista all'avanzata della nascente classe borghese (GARIN, 1964).

La trattatistica educativa, frutto di un processo di sistematizzazione delle riflessioni sull'educazione divulgate attraverso canali veicolari tradizionali, si contraddistinse per la ricchezza della progettualità pedagogica di cui era portatrice, animata dalla necessità di formare 
l'uomo nella completezza delle sue facoltà partendo dal riconoscimento della perfettibilità della sua natura. L'osservazione accurata e quotidiana delle inclinazioni mostrate dal neonato fin dai primi giorni di vita, «con l'intelletto e l'occhio desto per scrutarne l'indole», costituirono per gli umanisti il canale principale per intraprendere il più precocemente possibile indispensabili interventi educativi fondati su una pluralità di motivi: l'imitazione di buoni esempi (incarnati per primi dai genitori), la ricerca dell'eccellenza, la mitezza razionale, la cura della relazione fra educatore ed educando e della relazione fra pari, la preoccupazione di non turbare la serenità e la gioia dei più piccoli, l'attenzione al «vedere» e all' «ammirare», in una commistione di saperi dotti del corpo e dello spirito (BECCHI; JULIA, 1996a, p. 143-147).

Pier Paolo Vergerio il Vecchio (1370-1444) affermò nel primo trattatello sugli studi liberali con cui prese avvio l' «umanesimo educativo italiano» (CAGNOLATI, 2016), intitolato De ingenuis moribus et liberalibus studiis adulescentiae (1400-1402), che «è in quella verde età $[\ldots]$ che si devono gettare le fondamenta di tutto il nostro vivere, ed informare l'animo a virtù finché sia tenero e capace di ricevere qualunque impronta, la quale, come allora sarà fatta, durerà per tutta la nostra vita avvenire» (GARIN, 1964, p. 18). Anche Matteo Palmieri (14061475) raccomandava al «padre a cui sarà nato il figliuolo innanzi ad ogni altra cosa abbia di lui perfetta speranza». Il problema di avviare un'educazione dalla nascita era ormai ineludibile, poiché nei figli non si riponeva solamente il senso di un'immortalità concreta e terrena, ma anche la responsabilità verso il futuro degli uomini e della società (GARIN, 1976, p. 12). Il piccino iniziò ad essere sempre più considerato come un soggetto e non più una figura precaria, posto al centro di una nuova modalità di narrazione dell'educazione all'interno della cosiddetta «letteratura di famiglia», costituita da libri di famiglia, ricordanze, memorie, epistolari. Parallelamente, si verificò anche la diffusione di una trattatistica medica interessata a fornire indicazioni terapeutiche e di puericultura per piccoli e piccolissimi, come nei casi del Libellus de Egritudinibus infantium di Paolo Bagellardo e del Regimen des jungen Kinder di Bartholomaeus Metzinger (BECCHI; JULIA, 1996a, p. 122-123).

Uno dei contributi di riflessione più originali dell'Umanesimo italiano fu quello dell'architetto e letterato Leon Battista Alberti (1401-1472), simbolo dell' 'uomo universale», che nel trattato Libri Familie (= Libri della famiglia) - scritto in volgare e suddiviso in quattro libri, di cui tre redatti fra il 1432 e il 1434 - presentò una sintesi compiuta della prospettiva elaborata sino a quel momento dalla paideia umanistica sui temi della famiglia, della paternità e dell'educazione dei figli, a partire dalla centralità riconosciuta alla natura "propria" del bambino e dal ruolo protagonistico assegnato ai genitori nel porre le basi del suo percorso educativo fin dall'ambiente domestico. Il suo discorso ruotava attorno al riconoscimento della natura come migliore guida e maestra di tutte le cose, con la quale l'uomo avrebbe potuto costruire un rapporto armonico per mezzo dell'educazione, a partire dall'unione coniugale e dalla cura della prole (ALBERTI, 1994, p. 128-129).

Fra le prime incombenze del neo-padre, Alberti poneva la scelta accurata del nome del neonato, come gesto finalizzato alla costruzione dell'identità della persona, e la registrazione della data di nascita, consuetudine che fino ad allora non era presente nelle famiglie, nemmeno in quelle altolocate, ma che richiamava l'importanza della scrittura per la classe sociale mercantile in ascesa (ALBERTI, 1994, p. 146). Alberti fu attento ad affrontare il rapporto intergenerazionale fra padri e figli, analizzandolo alla luce di un'inedita idea di paternitas, caratterizzata dalla sollecitudine nelle cure, dal piacere e dalla gioia di allevare i figli, tipica del nuovo corso umanistico (HAAS, 1998, p. 1-15). Il ruolo del padre di famiglia non poteva più consistere soltanto nel dovere di riempire il granaio di casa e la culla, cioè di pensare al sostentamento economico e alla riproduzione, ma nell'assunzione di un nuovo profilo eticocomportamentale, politico e civile, in linea con quello veicolato dalla trattatistica "economica" di Età moderna (FRIGO, 1985, p. 10). 
Nel più ampio contesto dell'Umanesimo europeo, il richiamo ai doveri educativi del padre nei confronti dei figli rappresentò un paradigma educativo e culturale di natura idealtipica, pensato in funzione di stimolo culturale (GARIN, 1976). Si pensi a Erasmo da Rotterdam (1466-1536) che, nel modello pedagogico-morale dello speculum principis, propose un percorso di educazione liberale del sovrano dal primo vagito fino alla conquista della piena maturità del lógos, mostrando pari attenzione sia al portato della tradizione filosofica e pedagogica del mondo greco, ellenistico, latino e cristiano, sia ad una dimensione "concreta" attinente le implicazioni moderne della competenza politica del princeps (ERASMO, 1992, p. 18). La centralità riconosciuta al legame fra natura e cultura servì ad Erasmo per ribadire la necessità di avviare dalla nascita un'educazione non corruttrice, fondata sull'osservazione sistematica delle attitudini naturali del piccino e su un'idea di esercizio della paternità in senso educativo. Nella Declamatio de pueris statim ac liberaliter instituendis (1529), delineò il profilo di una nuova paideia, il cui fulcro era costituito da un'educazione concepita come percorso volto a mettere il neonato nelle condizioni migliori per esercitare la sua mente capace di apprendere, unico fra tutti i cuccioli di animali a possedere il lógos e, in quanto tale, dotato di una ricettività e di una capacità di assorbimento che rendevano indispensabile nutrire la sua anima con il "nettare" del sapere. Anche in Erasmo, il richiamo alla responsabilità educativa genitoriale a fronte dei pericoli della negligenza e dell'incuria, o dei drammi dell'infanticidio e dell'esposizione, si innestava su una visione del figlio come bene supremo: «quando la natura ti concede un figlio, ti affida un ammasso di carne grezza. È tuo dovere plasmare nel modo migliore una materia docile e duttile. Se sbagli ottieni una bestia, se sei avveduto ottieni, per così dire, un essere divino» (ERASMO, 1989, p. 106).

Una sensibilità simile era presente anche in un altro protagonista dell'Umanesimo europeo, Juan Luis Vives (1492-1540), che nella sua peculiare attenzione nei confronti dell'educazione della buona donna cristiana pose l'accento sul primato della funzione materna, in una cornice pedagogica volta a riconoscere il carattere educativo naturale dell'ambiente domestico e una visione rigorosa della vita delle bambine fin da quando erano allattate al seno materno. L'allattamento era considerato una prima occasione di relazione educativa e l'amore genitoriale costituiva, anche per Vives, il principale fattore di promozione di un'educazione in grado di combattere i germi della corruzione della natura umana dovuti al peccato originale (VIVES, 2000, p. 53-54). Esplicita era la ripresa di modelli del passato, tratti da Le notti attiche (159-170 d.C.) di Aulo Gellio e da alcune Epistole di Girolamo (400-413 d.C.).

Non va, infine, dimenticato che la paideia umanistica, in ragione della natura universale dell'ideale antropocentrico, non mancò di interessarsi dei neonati provenienti dalle classi sociali meno elevate, soprattutto di quelli abbandonati tramite esposizione. Nei paesi del Sud Europa sorsero i primi brefotrofi, grazie all'impegno di alcune congregazioni religiose, mentre in quelli del Centro-Nord Europa, a seguito della riforma luterana, venne adottata un'altra forma di tutela sociale degli esposti, quella della ricerca della paternità, con il conseguente addebito all'intera comunità locale delle spese di mantenimento del piccolo, al fine di scoraggiare l'abbandono dei figli illegittimi. Diversi studi hanno rilevato che la diffusione dei brefotrofi, con la loro opera di assistenza e la segretezza garantita ai genitori intenzionati ad esporre i loro piccoli, incentivarono anziché disincentivare la pratica dell'abbandono, spesso anche di figli legittimi di famiglie povere non in grado di mantenerli, soprattutto nei periodi storici di maggiore crisi economica o politica (STONE, 1977, p. 133-300; CUNNINGHAM, 1995, p. 114-119; POLENGHI, 2001, p. 1-7). In ogni caso, va riconosciuto che grazie a queste pratiche furono prese gradualmente le distanze dal carattere elitario della paideia greco-romana per aprire le porte alla modernità, sul duplice piano dell'assistenza degli esposti e dell'introduzione di forme di disciplinamento corporeo finalizzate alla correzione morale, che sarebbero state particolarmente esaltate dall' Ancien Régime (BECCHI; JULIA, 1996a, p. 143). Si può, pertanto, affermare che i due fenomeni 
furono fra loro intrecciati, poiché la progressiva diffusione di una nuova idea di neonato portò con sé l'avvio del processo di istituzionalizzazione dell'infanzia abbandonata e, nel contempo, la ricerca per chi sarebbe sopravvissuto alle insidie della prima infanzia di un percorso di vita il più precocemente autonomo possibile.

\section{Fra luteranesimo e riforma cattolica}

L" "uragano" provocato sul piano religioso, politico, culturale, sociale e pedagogico dall'opera riformatrice di Martin Lutero (1483-1546), che si suole far iniziare con la presunta affissione di 95 tesi al portone della cattedrale tedesca di Wittenberg dopo i vespri del 31 ottobre 1517, ebbe immediate conseguenze anche nell'ambito dei rapporti familiari, con lo sviluppo del fenomeno della spiritualization of the household, in ragione del quale ogni famiglia era chiamata ad assumere il ruolo di microcosmo spirituale della Chiesa luterana e dello Stato, come "vivaio" in cui coltivare le future generazioni pronte a servire la Chiesa e lo Stato (HILL, 1964, p. 443-481). L'accento posto sulla procreazione, quale dovere primario degli sposi, non comportò di per sé una maggiore valorizzazione dell'infanzia, considerata come «fonte di ansia e di gioie», nonostante ogni nuova nascita venisse festeggiata come un evento semi-pubblico (CUNNINGHAM, 1995, p. 46-47). Il neonato era investito di numerose aspettative, legate all'importanza di procurare la sua buona educazione mediante un'accorta vigilanza paterna, per garantirgli la conquista della salvezza eterna e mantenere il buon ordine sociale, religioso e statuale, secondo quanto illustrato nei catechismi a stampa. Era, pertanto, da reputarsi peccato grave abbandonare e disonorare le nobili anime dei piccini, secondo l'insegnamento espresso da Lutero in vari discorsi pubblici, come quello intitolato An die Ratsherren aller Städte deutschen Landes, dass sie christliche Schulen aufrichten und halten sollen (1524) (LUTERO, 1990, p. 37-39).

Il pastore moravo Jan Amos Comenio (1592-1670), nella prima metà del XVII secolo, sistematizzò questi principi in una teoria organica dell'educazione sotto forma di pedagogia, pensata come disciplina autonoma dai saperi filosofici, teologici, letterari che fino ad allora ne avevano costituito il principale terreno di espressione. Nell'affermare il principio di istruzione universale, posto al centro della sua Didactica Magna (1628), e in continuità con le nuove istanze di ricerca di un metodo universale promosse dalla rivoluzione scientifica seicentesca, si premurò di delineare un sistema educativo da 0 a 24 anni, che vedeva coinvolta la fascia d'età neonatale all'interno della cosiddetta Schola Infantiae (o Schola Materni Gremii), coincidente con l'ambiente domestico, quale migliore contesto per avviare un'educazione secondo natura dei più piccoli, in quanto arbusculas Dei (COMENIO, 1993, p. 446-464).

Da parte sua, il coevo processo di rinnovamento cattolico guidato dal papato, che trovò nel Concilio ecumenico di Trento (1545-1563) una delle sue massime espressioni, puntò parimenti ad una spiritualizzazione della vita domestica con i decreti dogmatici e disciplinari concernenti il sacramento del matrimonio. Quest'ultimo continuò, però, ad essere concepito come un vincolo indissolubile che legava due persone sulla base delle scelte compiute dai loro genitori, ancora più stringenti se si apparteneva a famiglie nobili o alto-borghesi, cosa che in sé non favoriva la fedeltà coniugale. Forti furono la condanna sociale e l'intolleranza nei confronti delle madri nubili e dei figli illegittimi (POLENGHI, 2001, p. 7). Anche la pubblicistica cattolica dell'epoca pose al centro il tema della responsabilità genitoriale nell'educazione dei più piccoli; in particolare, $\mathrm{i}$ catechismi e i manuali per i confessori si concentrarono attorno al rispetto del IV comandamento «onora il padre e la madre» - come richiamo al reciproco amore fra genitori e figli, in nome di un'inedita concezione della famiglia come «luogo d'incontro degli affetti» (CUNNINGHAM, 1995, p. 54-58). In Italia, la diffusione di tali principi in ambito educativo e familiare si avvantaggiò del contributo offerto dal cardinale Silvio Antoniano 
(1540-1603), collaboratore di papa Pio IV e amico di Carlo Borromeo, che con i Tre libri dell'educatione christiana dei figliuoli (1584), approvati dal vescovo di Verona Agostino Valier, delineò un progetto pedagogico di promozione dell'istruzione dei fanciulli ai costumi cristiani a partire dall'ambiente domestico (SANI, 2006, p. 64-65). Battezzato non appena nato, ogni figlio doveva essere sorvegliato e vigilato fin dal primo istante di vita, evitando di lasciarlo da solo vicino al fuoco, o di soffocarlo inavvertitamente nel letto dove dormiva con gli adulti. La disciplina del suo corpo, ottenuta in primis con la fasciatura, doveva procedere di pari passo con quella dell'anima, per il legame inscindibile fra queste due dimensioni della persona umana. Per questo motivo, l'avvio di una buona educazione passava attraverso il primo nutrimento dell'infante, con l'allattamento al seno della madre, in nome di una legge di natura che all'epoca risultava ancora assai poco rispettata, soprattutto dalle nobildonne, che preferivano affidare i loro neonati alle balie (ANTONIANO, 2010, p. 932-934). Questo tema, fra i più trattati fin dall'Antichità, assunse nell'Età del rinnovamento cattolico un particolare accento morale, strettamente legato alla continenza sessuale dei coniugi (GOODY, 1983, p. 33-47).

\section{Gli sviluppi nel pieno dell'Età moderna}

Per affrontare gli importanti passaggi che, nel pieno dell'Età moderna, condussero ad una progressiva "presa di coscienza" del cosiddetto «sentimento dell'infanzia» (ARIÈS, 1960, pp. 5155), occorre richiamare le conseguenze sul piano sociale e culturale della rivoluzione scientifica seicentesca, con l'affermazione del primato dell'esperienza e della ragione nella ricerca di leggi universali, in campo igienico, fisiologico e educativo. Si iniziò a studiare la natura del fanciullo (maschio e, soprattutto, primogenito) fin dalla nascita, osservandone i giochi, le parole, i gusti, le passioni, per conoscerne il temperamento e dedurne le migliori strategie educative per intervenire in direzione del suo futuro destino personale e professionale (BECCHI; JULIA, 1996a, p. 285). Si richiama, a tal proposito, la pratica sempre più diffusa delle Mémoires scritte da nobiluomini o nobildonne dell'epoca, a partire dall'osservazione quotidiana di neonati e infanti: un esempio interessante, che diede vita ad un nuovo "genere" letterario e ad un vero e proprio habitus educativo, fu il Journal de Jean Héroard, redatto dal medico Héroard come «diario della salute» del futuro sovrano francese Luigi XIII (HEROARD, 1989).

La progressiva diffusione della corrente filosofica dell'empirismo diede un ulteriore contributo alla "messa a fuoco" del neonato, sia nell'Inghilterra rivoluzionaria con John Locke (1632-1704), sia nella Francia prerivoluzionaria di François de Salignac de la Mothe-Fénelon (1651-1715), entrambi impegnati nel costruire un rapporto di interdipendenza fra la formalizzazione di un sapere pedagogico dall'età neonatale e l'affermazione del processo di educazione individuale borghese.

La formazione iniziale in campo medico di Locke, impregnata di nuovi modelli di studio del corpo e della psiche fondati sull'osservazione di dati empiricamente rilevabili, si coniugò con un'istanza di natura politica, legata alle esigenze della classe borghese in ascesa e alla formazione dei cittadini della nascente monarchia parlamentare britannica, e con il rispetto della morale puritana. Una sintesi del suo pensiero più compiutamente pedagogico era rinvenibile nell'opera Some thoughts concerning education, scritta fra il 1684 e il 1691 su richiesta dell'amico Edward Clarke, per avere suggerimenti su come educare il proprio figlio. Il nucleo portante era costituito dall'avvio precoce di pratiche di cura e di educazione, rivolte ad un neonato considerato come una tabula rasa (LOCKE, 1937, p. 29-30), sulla quale l'esperienza avrebbe introdotto e sovrascritto le conoscenze attraverso le impressioni ricevute dai cinque sensi, secondo quanto illustrato da Locke nella sua più importante opera filosofica, An essay concerning human understanding (1689). La cura del corpo del neonato era pensata per garantire quei caratteri di robustezza, forza e resistenza senza i quali non sarebbe stato possibile 
preparare un buon terreno di coltura per l'educazione, secondo la cosiddetta "teoria dell'indurimento" ispirata alla paideia spartana. Esplicito fu il riferimento all'individualità dei più piccoli, riconosciuti nei loro bisogni peculiari, in contrasto con le tradizionali visioni adultistiche o adultocentriche. Anche i figli delle classi benestanti, al pari di quelli dei contadini, dovevano poter vivere all'aria libera, con un'alternanza equilibrata di esercizio fisico e riposo, nella perfetta sobrietà, vestiti con abiti adeguati (né troppo caldi, né troppo stretti), con il capo e i piedi non troppo riparati dal freddo, senza far uso di vino o di alcoolici, e nemmeno di farmaci, se non strettamente necessari (LOCKE, 1937, p. 53-54). L'originalità della prospettiva lockiana consistette, prevalentemente, nella rilettura in prospettiva empiristica della tesi ormai "classica" della coltivazione di buone abitudini fin dalla nascita, assumendo in questo modo l'acquisizione di habitus morali e culturali quale minimo comune denominatore di ogni intervento educativo pronto a fare di ogni uomo, in quanto essere razionale, il "padrone" di se stesso e del suo rapporto con gli altri, in nome del principio di self-government. Locke propose un'educazione di tipo pratico, fondata sul riconoscimento della natura imitatrice dei neonati definiti come «camaleonti» (LOCKE, 1937, p. 93), scevra da qualsiasi forma di punizione corporale o verbale, ma attenta allo sviluppo cognitivo attraverso il graduale affinamento della manualità con l'utilizzo di oggetti di uso comune.

Se Locke formulò i suoi pensieri sull'educazione destinandoli prioritariamente ad un fanciullo di sesso maschile, François de Salignac de la Mothe-Fénelon, meglio noto come Fénelon (1651-1715), ebbe un ruolo di primo piano nel panorama pedagogico internazionale fra Seicento e Settecento nel sostenere l'importanza di educare le fanciulle fin dalla culla, per combattere i rischi di una cattiva formazione, che si sarebbero poi riverberati sul piano della conservazione della solidità e della bontà delle relazioni familiari, una volta giunte al matrimonio e alla maternità (FENELON, 1963, p. 59). Nel Traité de l'éducation des filles (1687), scritto su sollecitazione della duchessa di Beauvilliers per l'educazione delle sue otto figlie e rivisto prima della pubblicazione per indirizzarlo alle madri e alle governanti, Fénelon prese spunto dal suo magistero pedagogico sviluppato nel corso del tempo come precettore del duca di Borgogna e dalle riflessioni ricavate dalla lettura di Platone, Agostino, Malebranche, Cartesio. Questo intreccio di ispirazioni fece emergere una rinnovata idea di bambino, non più concepito come un essere deficitario, ma come un essere in evoluzione stadiale dalla nascita, e il riconoscimento del primato dell'autoeducazione da promuovere attraverso le "lezioni di cose" offerte dalla vita quotidiana (FENELON, 1963, p. 60-66).

La donna-madre rappresentava per Fénelon la figura ideale di educatrice della prima infanzia, in quanto previdente, laboriosa, semplice, sincera e sensibile, dedita al marito, all'amministrazione domestica e all'educazione dei figli, garante dell'equilibrio e della serenità della società intera. Una delle strategie più efficaci suggerita alle madri per conoscere la natura dei loro figli, nei suoi caratteri di fisicità e suggestionabilità, consistette nella pratica quotidiana dell'osservazione e di un'azione educativa capace di far leva sull'immaginazione e sul sentimento, quali motori della vita spirituale e intellettuale dei neonati (PANCERA, 1991, p. 49-53). Risultava fondamentale orientare in modo indiretto, «per mezzo della ragione fin dove si può», le loro prime impressioni, con parole accompagnate da adeguati toni e gesti, per maturare una naturale inclinazione ad intrattenersi con le persone buone e dabbene, e ad allontanarsi da persone sconsigliabili perché viste in atteggiamenti sconvenienti o collerici.

Il richiamo reiterato di Fénelon all'efficacia educativa dei buoni esempi, corroborato dal riconoscimento della "plasticità" cerebrale e della "ricettività" dell'intelligenza infantile, testimoniava il persistere di una tradizione educativa centrata sull'imitazione quale canale prioritario di apprendimento per i più piccoli. La sua negazione di ogni innatismo - al pari di Locke, con cui condivise anche l'attenzione alla salute e alla corretta alimentazione - lo condusse ad affermare la tesi classica dell'infante come «cera molle» modellata dalle impressioni ricavate dall'esperienza sensibile. Non si 
può non riconoscere in queste tesi di Fénelon l'anticipazione di alcune dimensioni costitutive della pedagogia rousseauiana, in particolare l'idea di natura umana come physis fin dalla nascita, la pratica di un'educazione indiretta contro il pedantismo e il riconoscimento dell'esperienza come prima maestra (SCAGLIA, 2020, p. 33).

\section{La rivoluzione pedagogica di Rousseau}

L'Età dei Lumi vide un'accentuazione delle novità in tema di educazione neonatale nelle famiglie benestanti, con un maggiore rilievo educativo riconosciuto alla figura materna e la promozione di un'educazione individualizzata nello spazio domestico, grazie alla progressiva avanzata fra le corti europee e il relativo entourage intellettuale di una spiccata sensibilità nei confronti delle caratteristiche e delle esigenze dei più piccoli, in stretta connessione con il processo di «privatizzazione degli affetti» dovuto alla prima diffusione, soprattutto nell'Europa centro-settentrionale, della struttura familiare nucleare (SHORTER, 1975; CUNNINGHAM, 1995). Questo non impedì la persistenza, soprattutto nella cultura borghese, di forme di punizione fisica e psicologica volte al controllo e al contenimento psicofisico dei più piccoli (RUTSCHKY, 2015, p. 171). Fra le classi dirigenti francesi e inglesi si iniziò ad attestare una graduale riduzione del tasso di mortalità infantile, grazie alla felice combinazione di una serie di fattori: lo sviluppo di profondi cambiamenti culturali ed educativi, che aprirono la strada ad un'inedita attenzione nei confronti dei bambini sul piano affettivo e dell'istruzione; la diffusione di nuove pratiche mediche e igieniche; l'incentivazione da parte dei medici dell'allattamento al seno materno e dell'abolizione della fasciatura; il ricorso sistematico alla contraccezione, diffusasi inizialmente fra i notabili residenti nelle zone urbane (BECCHI; JULIA, 1996b, p. 46-47). Gli spazi domestici delle famiglie benestanti subirono in quel periodo importanti modifiche, che condussero all'introduzione nei decenni successivi della "cameretta" dei bambini, che richiamava la centralità della casa come simbolo di un nuovo ordine interiore, politico e sociale (COVATO, 2004, p. 76). L'immagine della madre come donna felice di dedicarsi alla cura dei propri figli e alla tenuta dell'intimità familiare divenne sempre più preponderante.

Occorre, però, evitare - come raccomandato da Dominique Julia - di considerare questa mutazione di valori, habitus e vissuti come la diretta fonte ispiratrice della teoria pedagogica illustrata da Jean Jacques Rousseau (1712-1778) nel romanzo Émile o de l'éducation (1762), poiché il suo intento non fu quello di farsi "portavoce" di tali istanze, bensì di proporre una nuova prospettiva pedagogica fondata sulla centralità del principio metafisico della physis, in aperto contrasto con le prassi educative tradizionali (BECCHI; JULIA, 1996b, p. 65). Frutto di «vent'anni di meditazione e tre ore di lavoro», l'Émile si presentò ai lettori come un «romanzo pedagogico» in cui si intrecciarono differenti generi letterari, allo scopo di superare - polemizzando - le comuni forme del trattato pedagogico, che rischiavano di farne un prontuario per migliorare la cura e l'allevamento dei piccini. Il lettore si trovava, così, di fronte ad una concezione di educazione secondo natura, nella sua duplice tensione etica e pedagogica. Con questa affermazione, carica di una pluralità di motivi, Rousseau inaugurò una nuova "stagione" nella riflessione pedagogica occidentale, grazie al fatto che il riconoscimento della centralità del fanciullo nei processi educativi (puerocentrismo) si accompagnò a quello della perfettibilità umana quale condizione di possibilità - anziché condizione ostativa - dell'educazione (ROUSSEAU, 2016, p. 13-15).

Si spiega, così, la sua attenzione peculiare nei confronti delle esigenze educative e psicologiche dell'infanzia fin dalla nascita, in linea con la maturazione del «sentimento dell'infanzia», ma sempre nell'ambito di una più generale intenzionalità filosofica e pedagogica. L'età neonatale, al centro del I libro dell'Émile dedicato all'educazione fra 0 e 5 
anni, assunse una nuova significazione, poiché venne innalzata a condizione ideale nella quale la natura dell'uomo - da intendersi nel senso classico filosofico di physis, e non nel senso di una natura biologico-fisiologica - si manifestava nell'integralità della sua bontà e come tale doveva essere coinvolta in una forma di educazione "personalizzata", che ne rispettasse i caratteri, i tempi di sviluppo, le modalità di manifestazione (ROUSSEAU, 2016, p. 72-93).

Nell'Émile, Rousseau intese descrivere le condizioni ideali per promuovere un'educazione come «arte del vivere» e lo fece avendo in mente l'insegnamento di Montaigne, a proposito della lotta a quei condizionamenti - come la fasciatura dei neonati e il baliatico - che ostacolavano un'educazione secondo natura, contribuendo al contenimento e alla repressione della libertà propria di ogni essere umano (MONTAIGNE, 1965, p. 404-405). Per questo motivo, nel laboratorio pedagogico dell'Émile la vita neonatale e la relazione madre-bambino offrirono le prime occasioni di riflessione educativa. Va premesso che la figura materna, esaltata quale «prima educatrice», risultò essere una "grande assente" nel romanzo, al contrario di quanto narrato nella Julie ou La nouvelle Héloüse a proposito delle cure e dell'impegno educativo di Julie nei confronti dei suoi tre figli, descritti nella III lettera contenuta nella V parte del volume. La scelta intrapresa nell'Émile era dovuta al fatto che nell'esperienza quotidiana, la madre altolocata si faceva supplire da nutrici e governanti, tanto da creare una serie di contraddizioni e una rottura di quell'unitarietà dell'azione educativa, che sola poteva guidare l'educazione infantile secondo natura (ROUSSEAU, 2016, p. 80-81).

Per questo motivo, Rousseau sostituì nella narrazione dell'Émile la figura materna con quella del gouverneur, incaricato di occuparsi della prima educazione sensoriale del neonato, fondata sui dati percepiti attraverso i cinque sensi, poiché l'esperienza era la fonte primaria dell'apprendimento infantile. Il neonato, lasciato libero dalle fasce, poteva costruire attraverso l'esplorazione sensoriale quell'indispensabile rapporto diretto ed immediato con la realtà esterna. Il piccolo per Rousseau nasceva capace di imparare, quindi ogni occasione per muoversi, o piangere, o esprimersi nella sua lingua naturale fatta di suoni e di gesti, era motivo di apprendimento e come tale andava favorita, non negata. La scelta degli oggetti da mostrare al bambino costituiva una strategia educativa in grado di garantirgli la libertà più autentica senza cadere nell'arbitrarietà, perché gli offriva la possibilità di esperire nuove sensazioni in un ordine adeguato, a sua misura (ROUSSEAU, 2016, p. 115).

\section{La nascita della pedagogia della madre fra Pestalozzi e Necker de Saussure}

La cultura di ispirazione rousseauiana si diffuse ben presto a macchia d'olio nelle corti e nelle principali città europee, compresa Zurigo, da tempo crocevia delle istanze dell'illuminismo francese filtrato dalla tradizione protestante, del patriottismo svizzero, dell'umanitarismo, del pietismo e della fisiocrazia. Fu in quel milieu, che il giovane Johann Heinrich Pestalozzi (1746-1827) lesse l'Émile e ne riprese alcuni principi sul piano della teoria pedagogica e della prassi educativa, non rinunciando però a riconoscere la funzione educativa della famiglia e il primato materno (POLENGHI, 2001, p. 16-19).

Fin dalle sue prime opere, come il romanzo pedagogico Lienhard und Gertrud: Ein Buch für das Volk (1781-1787), Pestalozzi mise in risalto l'agire educativo materno considerandolo un canale privilegiato per promuovere un'educazione secondo natura e per la rigenerazione morale e sociale del popolo, a partire dall'intimità dello spazio domestico. Anche all'interno del saggio Ueber Gesezgebung und Kindermort (1783), sostenne strenuamente il carattere naturale dell'amore materno, a fronte delle deviazioni provocate dai condizionamenti sociali e culturali. Pensato come intervento a difesa delle donne condannate a morte per infanticidio, consentì a Pestalozzi di richiamare l'importanza di trovare una soluzione a questo crimine attraverso una riforma sociale e legislativa, in cui venisse rivisto il concetto di responsabilità personale e, nel contempo, si facesse affidamento alle funzioni 
preventive dell'educazione (PESTALOZZI, 1999, p.5-57). Il neonato andava riconosciuto come possessore di germi invisibili da far crescere, una sorta di «bocciolo non ancora dischiuso» e di «tenero arboscello» affidato alle cure pazienti e alle attenzioni amorevoli della madre, ricettacolo della sua vita e perno attorno al quale ruotava la famiglia, prima sfera di vita dell'uomo e realtà intermedia fra il singolo e la comunità. La rivoluzione pedagogica soggiacente al pestalozzismo assunse i contorni di una «nuova rivoluzione protestante» in grado di riproporre i migliori fermenti del pensiero luterano sul piano educativo, grazie ad una felice sintesi fra l'istanza di universalizzazione dell'istruzione e dell'educazione sostenuta da Jan Amos Comenio e l'istanza rousseauiana dell'educazione personalizzata secondo natura, per giungere a promuovere un' «educazione domestica del popolo secondo natura» (SCAGLIA, 2020, p. 59).

Negli ultimi anni di insegnamento presso la scuola di Burgdorf, Pestalozzi decise di pubblicare accanto ad un volume dedicato al metodo di insegnamento intuitivo elementare, dal titolo Wie Gertrud Ihre Kinder Iehrt (1801), un testo pensato come guida teorico-pratica per le madri alle prese con l'educazione dei loro piccini. Si trattò di un progetto, realizzato solo in parte, con il Buch der Mütter oder Anleitung für Mütter ihre Kinder bemerken und reden zu lehren. Erstes heft uscito nel 1803, tradizionalmente segnalato come opera pestalozziana, ma in realtà redatto dal collaboratore Hermann Krüsi. Fin dall'introduzione, fu affermata l'importanza dell'azione educativa della madre nell'attivare al meglio i germi vitali dello sviluppo presenti nel bambino, attraverso la promozione delle capacità di osservare e parlare grazie al libero movimento e all'esplorazione della realtà circostante. La sua sollecitudine naturale le consentiva di agire nella maniera più idonea ed opportuna, per favorire una prima educazione dei sensi. Pestalozzi-Krüsi mostrarono, in questo modo, di aver compreso la lezione rousseauiana di un'educazione dalla nascita che, attraverso l'esperienza come prima "maestra" dell'uomo, stimolasse l'apprendimento del neonato, ancor prima che imparasse a parlare (ROUSSEAU, 2016, p. 113).

Con una serie di raccomandazioni aperte dall'esclamazione «Und Mütter!», PestalozziKrüsi condussero le destinatarie ideali del volumetto lungo la strada tracciata dalla Provvidenza, grazie al suggerimento di mezzi «semplici», «facili» da utilizzare e «appropriati» in vista del perfezionamento intellettuale e morale dei loro piccoli. L'idea di sviluppo che animava la loro proposta pedagogica non era riducibile alla mera crescita fisiologico-biologica del corpo e della mente del neonato, bensì in senso ampio e integrale alla formazione di tutte le sue dimensioni, a partire dalla constatazione che l'intervento materno, quale guida esterna, potesse aiutare il piccolo ad imparare gradualmente da solo a dirigere i primi sforzi della sua attenzione al corpo, alla mente e al cuore (PESTALOZZI, 1803, p. V-XIV).

La natura di Elementar Buch del testo rese, però, necessario ricondurlo ad una specifica cornice pedagogica di riferimento, per coglierne al meglio le novità, fissate nei loro capisaldi all'interno di 34 lettere inviate da Pestalozzi all'amico inglese James Pierpoint Greaves fra il $1^{\circ}$ ottobre 1818 e il 12 maggio 1819 , poi raccolte nell'opera Mutter und Kind pubblicata nel 1827. La cura materna delle prime manifestazioni dell'animo infantile venne presentata come una sorta di conditio sine qua non per avviare il processo di rigenerazione morale e sociale del popolo (PESTALOZZI, 1927, p. 14-15). Il legame madre-neonato in tutta la sua naturalità confermata dall'esperienza, ma non per questo in contraddizione con lo spirito cristiano - era uno "specchio" di quel sentimento primordiale di amore e fiducia che legava ogni uomo al suo Creatore e che, sul piano strettamente educativo, vedeva la madre come colei che faceva da "sentinella" nell'osservare e cogliere, con coraggio e umiltà, le prime espressioni dello sviluppo fisico, sensoriale, razionale e spirituale del neonato. La figura materna, investita di significati pedagogici, socio-politici e religiosi, risultò in questo modo protagonista di una progettualità educativa che richiedeva l'esercizio sistematico, costante ma non per questo artificioso, di un «amore pensoso», cioè di un amore regolato "con" e "dalla" riflessione sulla natura dei propri 
doveri educativi volti a condurre al bene i propri figli (PESTALOZZI, 1927, p. 17). Per ogni madre era prospettato un compito di elevazione spirituale che, partendo dall'osservazione della gratitudine e della naturale sympatheia espresse dal neonato nei confronti delle sue cure, trovava via via compimento nel raggiungimento e nel mantenimento del benessere fisico del bambino e della sua «tranquillità morale e spirituale», a testimonianza del pieno ed integrale svolgimento della sua natura nel presente e, soprattutto, in vista del futuro (PESTALOZZI, 1927, p. 34-35).

Accanto alla figura di Pestalozzi, nel panorama pedagogico europeo fra illuminismo e romanticismo si formò anche il profilo intellettuale di Albertine Necker de Saussure (17661841), artefice di una pedagogia del neonato fondata sui principi della benevolenza reciproca, della giusta serietà e dell'ordine. Figlia di uno scienziato e filosofo protestante, Orazio Benedetto de Saussure, crebbe nel circuito culturale delle famiglie dell'alta borghesia ginevrina, vivendo a cavallo di tre grandi passaggi storici: la rivoluzione francese, il periodo napoleonico e la successiva restaurazione del potere dei sovrani europei (SCAGLIA, 2020, p. 64).

Il contributo di Albertina Necker de Saussure, che per buona parte della sua vita intrecciò lo studio al ruolo di madre scrupolosa impegnata nell'educazione dei suoi quattro figli, risultò centrale nell'ambito della pedagogia post-rousseauiana grazie alla sua principale opera, dal titolo L'éducation progressive, ou Étude du course de la vie (1828-1838). Dalla sua lettura emerge l'interesse ad interloquire in maniera critico-riflessiva con il portato più originale della pedagogia rousseauiana, richiamato dall'utilizzo dell'espressione éducation progressive, intesa dall'autrice come processo di autoeducazione della persona umana, interpretato alla luce di una teleologia e di un'antropologia compiutamente cristiane (NECKER DE SAUSSURE, 1940).

Dagli appunti raccolti nei suoi quaderni trapelava una conoscenza approfondita degli autori post-rousseauiani, come Pestalozzi, di cui condivise la visione dell'ambiente domestico come miglior terreno in cui promuovere l'educazione naturale e il ruolo della madre come prima educatrice, senza però affrontare il tema della rigenerazione umana e sociale del popolo. Per poter realizzare il lavorio di auto-perfezionamento continuo al centro della sua teleologia, la studiosa ginevrina raccomandò di affiancare alle forme di «educazione accidentale», che pur accompagnavano l'uomo fin dalla prima età, una forma di «educazione premeditata» (NECKER DE SAUSSURE, 1940, p. 61).

L'attenzione e la cura con cui la Necker de Saussure affrontò l'educazione del neonato nei primi tre libri de L'éducation progressive celavano dietro di sé un interesse epistemologico, e non solo teleologico-metodologico, nei confronti dell'educazione. Fra le prime questioni affrontate vi era la differenza esistente fra le cure materne negli animali e le cure materne negli uomini, dalla quale era possibile rilevare quanto l'arte dell'educazione umana non fosse il prodotto di un istinto-guida. La migliore strategia metodologica suggerita dall'autrice consisteva nel praticare un'osservazione costante e accurata dei neonati e dei fanciulli, indispensabile per poter studiare il loro sviluppo nelle sue molteplici dimensioni e sfumature, che vedevano un contestuale - e non succedaneo - emergere dello sviluppo dei sensi, della formazione delle funzioni cognitive e della formazione del linguaggio. La Necker de Saussure non nascose le difficoltà dell'educazione, legate al fatto che il piccino appariva come «una specie di selvaggetto, un po' incivilito esteriormente», che aveva un modo di sentire, legare e confrontare le idee tutto suo, del tutto differente da quello degli adulti. Risultava fondamentale far leva sulle dimensioni emotive e sensoriali particolarmente attive ai primordi della vita umana, incanalandole verso l'espressione di una benevolenza e di una calma abituali, che nulla avessero a che vedere con manifestazioni come i capricci, dovute quasi sempre ad una speranza delusa, o ad una sofferenza sentita, o a sensazioni troppo vivamente eccitate (NECKER DE SAUSSURE, 1940, p. 98-147).

La Necker de Saussure mostrò anche un particolare interesse per lo studio della genealogia dell'intenzionalità nella persona umana, al fine di promuovere una migliore 
educazione secondo natura. Le sue considerazioni presero avvio dal mistero e dalle sofferenze della nascita, per giungere allo sviluppo dell'istinto di suzione, fino alle prime risposte del neonato alle sensazioni interne, da considerarsi come avvio del suo processo di autoeducazione. Grande attenzione dedicò anche alla genesi del linguaggio naturale, già studiato da Rousseau, e alla capacità di manipolazione. L'uso sempre più preciso e articolato della mano corrispondeva, sul piano dello sviluppo cognitivo, al processo di progressiva concorrenza della vista e del tatto nel fornire un'idea unitaria dello stesso oggetto, poiché il bambino sapeva ormai assegnare la maggior parte delle sue impressioni alle loro cause, rettificava gli eventuali errori della vista per mezzo del tatto e non si ingannava più sulla distanza degli oggetti posti accanto a lui (NECKER DE SAUSSURE, 1940, p. 105).

\section{Il fröbelismo}

La rivoluzione pedagogica pestalozziana trovò ulteriori risonanze nell'opera dello studioso tedesco Friedrich Fröbel (1782-1852), che a partire dalla lettura del Buch der Mütter iniziò a gettare i pilastri di una vera e propria pedagogia della prima infanzia, spinto da «ciò che di più elevato l'animo amoroso del Pestalozzi aveva potuto dare al genere umano per l'allevamento dei fanciulli» (PRÜFER, 1927, p. 24). Di particolare rilievo fu la sua riflessione attorno alla centralità dell'osservazione, nel favorire una conoscenza profonda del mondo esterno, e della lingua come rappresentazione del mondo. Volle affidare la «prima scuola dell'uomo» alla madre, in quanto responsabile della costruzione del futuro edificio culturale di suo figlio attraverso l'osservazione del mondo esterno e l'insegnamento della lingua. Per questo motivo, mise a punto esercizi di osservazione pensati per stimolare fin dall'età neonatale la capacità di discriminazione, intuizione ed espressione all'interno di una cornice pedagogicodidattica più ricca ed elaborata di quella soggiacente al Buch der Mütter, in quanto fondata sulla cosiddetta «legge sferica», capace di regolare l'unità esistente fra essere e divenire e che costituiva la base per la formazione spirituale degli uomini, secondo quanto da lui stesso delineato nell'opera Die Menschenerziehung (1826).

Cura materna e sviluppo infantile risultarono, pertanto, complementari e fondati sulla valorizzazione della naturale ed irrinunciabile «missione educativa» delle donne in famiglia, da espletarsi secondo le modalità più conformi ad essere una buona «madre cristiana», come Fröbel volle esprimere in diversi suoi appelli alle giovani in procinto di sposarsi. Il primato attribuito all'amore materno rispetto a quello paterno gli consentì di dare vita nel 1840 ad un'istituzione - la cosiddetta «scuola delle madri», o Kindergarten - in grado di concretizzare al meglio tali principi e porsi l'obiettivo di formare nuove professionalità dedicate all'educazione di fanciulle e fanciulli dalla nascita ai 6 anni (POLENGHI, 2001, p. 19-20; SCAGLIA, 2020, p. 102-112). Forte di questi risultati, nel 1844 pensò di predisporre un valido ausilio per "attrezzare" dal punto di vista pedagogico-didattico tutte le donne, in vista di una progressiva assunzione di consapevolezza del loro ruolo di «maternità educante»: si trattò di un «libro di famiglia» intitolato Mutter-und Koselieder. Dichtung und Bilder zur edlen Pflege des Kinderheitlebens: ein Familienbuch. Il lavoro venne concepito come guida teorico-pratica per le madri educatrici, a partire da una serie di osservazioni e di constatazioni effettuate da Fröbel stesso durante la sua collaborazione con Friederike Schmidt di Gera, dal 1840 al 1844. Ebbe la supervisione di Kohl per la parte musicale e di Unger per le illustrazioni.

La proposta culturale e pedagogica alle radici di quest'opera non può essere compresa nell'interezza dei suoi intenti se non la si riconduce all'idea di Kindergarten come «paradiso dell'infanzia», in cui far crescere i "teneri germogli" dei bambini. Il neonato era seguito nello sviluppo delle sue prime abilità, grazie al suggerimento di esperienze di movimento, discriminazione sensoriale, imitazione, percezione di svariati oggetti a partire dall'ambiente domestico (LASCARIDES; HINITZ, 2011, p. 104-105). Per esempio, la sua forza era esercitata 
favorendo il pestare con i piedi quanto capitava sott'occhio e l'afferrare ciò che la mano toccava, mentre la manifestazione dei primi sentimenti verso gli altri avveniva attraverso il sorriso e le sensazioni di piacere, provate quando il suo viso iniziava a volgersi verso la luce e l'aria (FRÖBEL, 1874, p. 9). La «parola materna» costituiva, in tutti questi casi, il principale veicolo per favorire l'interazione del piccino con il mondo esterno e la costruzione della sua visione della realtà. Del resto, il rapporto madre-figlio illustrato dall'iconografia a corredo del Mutter-und Koselieder lasciava intravedere uno spirito di «comunione continua con la libera natura», all'interno di un contesto rurale in cui, giorno dopo giorno, si svolgeva una crescita armonica dei piccini alla ricerca di un' «amorosa» continuità fra ordine naturale e ordine sovrannaturale, di cui era garante il ruolo educativo materno (SCAGLIA, 2020, p. 112-116).

L'originalità del contributo froebeliano risiedette nell'aver coniugato questi motivi più espressamente romantici e ottocenteschi con il riconoscimento di un inedito protagonismo educativo femminile, che di lì a poco avrebbe trovato conferma nei primi movimenti internazionali a sostegno dei diritti femminili e di una visione della donna, svincolata da un retaggio plurisecolare di condizionamenti familiari, sociali e culturali. Non per nulla, furono soprattutto donne di origine aristocratica o alto borghese a fungere da propagandiste del fröbelismo, come Berta von Marenholz-Bülow per l'area tedesca e più in generale europea, Elena Raffalovich Comparetti e Adele Levi della Vida per quella italiana, Elizaveta Vodovozova per quella russa, tanto da poter qualificare il loro impegno come una forma di spiritual motherhood (ALLEN, 1982).

Le aspirazioni emancipative di segno democratico che trovarono, in questo modo, una linea di convergenza con la pedagogia fröbeliana, si confrontarono anche con la diffusione nel più ampio contesto europeo delle prime istituzioni educative per la fascia d'età 3-6 anni - ispirate ad una «pedagogia del movimento, dell'amore, della gioia, dell'intuizione» presente nel modello della infant school di Robert Owen - e con una maggiore attenzione in campo medico e pedagogico alla piaga dell'esposizione infantile e della mortalità neonatale (BECCHI; JULIA, 1996b, p. 300). L'apertura in Francia delle prime crèche, alla metà degli anni Quaranta dell'Ottocento, rappresentò il tentativo di offrire un ricovero giornaliero e un'assistenza adeguata ai lattanti e agli svezzati fino al terzo anno di età, nell'ambito di un progetto politico e culturale di lotta al fenomeno dell'esposizione nelle aree urbane interessate dall'industrializzazione, a causa del sempre maggiore impiego di manodopera femminile (CAROLI, 2017, p. 3-39).

Con l'avvento del positivismo, la medicina divenne oggetto di un processo di «scientificizzazione-epistemologizzazione»e di «istituzionalizzazione-socializzazione», con l'assunzione di un ruolo di rilievo nel progresso delle scienze (anche umane) e nella promozione a livello sociale e politico di una nuova idea di salute e di neonato, accompagnata da azioni di prevenzione, profilassi, normalizzazione, controllo (CAMBI; ULIVIERI, 1988, p. 53-59). Nacque, in questo modo, un nesso sempre più stretto fra la medicina, l'ideologia borghese e l'impegno sociale, che comportò sul piano pedagogico una maggiore attenzione all'educabilità dei neonati e la necessità di formulare nuovi metodi pensati specificamente per loro (CAROLI, 2017, p. 106-110).

\section{Il primo Novecento: i contributi di Janusz Korczak e Maria Montessori}

L'avvento del XX secolo segnò in campo pedagogico la diffusione e il consolidamento in diverse zone dell'Europa e del Nord America del movimento dell'educazione nuova, la cui portata può essere descritta ricorrendo alla metafora deweyana della «rivoluzione copernicana». La nascita della psicoanalisi, lo sviluppo di prospettive pedagogiche alternative al tardopositivismo, il movimento internazionale di emancipazione femminile, che vide fra le sue maggiori protagoniste la scrittrice svedese Ellen Key e la pedagogista italiana Maria 
Montessori, contribuirono a tali cambiamenti. In particolare, Ellen Key mise al centro del volume di successo The century of the child (1900) la creazione di un processo di reciproca interdipendenza fra alcuni temi cari alla cultura femminile - come il rapporto fra sfera pubblica e sfera privata, la maternità intesa come social motherhood, l'autonomia individuale - e l'impegno per il riconoscimento dei diritti dei più piccoli, in linea con l'avanzata del concetto novecentesco di diritto (PIRONI, 2010, p. 4-9).

Uno spazio di rilievo assunsero, in tale contesto, alcuni autori europei di fama internazionale come Janusz Korczak (1878-1942) e Maria Montessori (1870-1952), entrambi con una formazione iniziale di medici. Essi non furono, senz'altro, i primi e gli unici ad occuparsi dello sviluppo psichico del neonato e della sua educazione, così come del carattere formativo dell'ambiente, ma mostrarono più di altri di aver tesaurizzato le scoperte e le innovazioni introdotte dai più recenti studi nei diversi campi delle scienze umane e della medicina. Il loro principale tratto di distinzione consistette nel continuare a riporre fiducia nella funzione imprescindibile e insostituibile della pedagogia, come scienza in grado di studiare e promuovere un'educazione dalla nascita grazie ad un intervento educativo indiretto e "su misura", in grado di far comprendere al meglio la natura del neonato e di operare a sua difesa, riconoscendone i diritti e valorizzando sul piano pedagogico ogni suo gesto, come espressione di una personalità in costruzione (SCAGLIA, 2020, p. 150).

Janusz Korczak rappresentò una figura poliedrica nel campo del riconoscimento dei diritti dell'infanzia e della divulgazione di una pedagogia puerocentrica. Dopo aver aperto la Dom Sierot a Varsavia nel 1913, volle indagare in prospettiva pedagogica gli aspetti «misteriosi» ed intimi della vita intrauterina e di quella neonatale, per mostrare la singolarità del bambino fin da quando era un feto e il rispetto a lui dovuto. Ne Il bambino in famiglia (1918-1920), affermò che il rapporto simbiotico vissuto con la madre non doveva privarlo del diritto ad essere riconosciuto come una realtà a sé stante e indipendente, che nella comune esperienza dolorosa del parto si sarebbe fatta strada con forza e determinatezza nella vita, dominata da un bisogno di indipendenza - «voglio vivere la mia vita» - a cui la madre avrebbe risposto affermativamente con il monito: «vivi la tua vita!» (KORCZAK, 1997, p. 19-25).

Fin dalla nascita, il bambino si presentava come una «pergamena fittamente ricoperta di minuti geroglifici», dei quali l'adulto era in grado di decifrare solo una parte. Quello che contava più di tutto, però, era la consapevolezza di come non potesse esistere una buona educazione del piccino senza la sua partecipazione attiva, facendo leva con fiducia sulle naturali capacità genitoriali. Solamente passando dall'esperienza delle notti insonni, dei pianti e delle malattie infantili - più che dai libri e dai consigli offerti da altri - sarebbe stato possibile per ogni genitore vivere interiormente una «profonda rivoluzione spirituale», capace di insegnare ad agire nelle condizioni date (KORCZAK, 1997, p. 30). Fondamentale risultava essere «l'intuizione del cuore di una mamma, la chiaroveggenza fondata sulla volontà di indagare, sulla vigilanza della mente, sulla limpidità del sentimento», quasi a riecheggiare la concezione pestalozziana dell'amore materno come «amore pensoso».

Fra i primi terreni di messa alla prova di questo atteggiamento educativo vi era l'allattamento al seno, inteso da Korczak come una prosecuzione della gravidanza, in linea con il pensiero antico che considerava il latte materno come sangue della madre trasformatosi lungo il passaggio dall'utero ai seni, ma anche con quello contemporaneo che stava scoprendo la capacità di autoregolazione neonatale del ritmo e dei tempi delle poppate, portando così le madri a "calibrarsi" sulle esigenze del piccino. Elementi come la temperatura della stanza e la quantità di latte e di acqua andavano considerati come dei veri e propri fattori educativi, e non solo igienici, per la loro diretta rispondenza alle esigenze della vita psichica dei più piccoli. Solamente partendo da queste consapevolezze, per Korczak sarebbe stato possibile costruire le basi di un'educazione neonatale all'insegna dei principi di libertà e di rispetto di ogni bambino (KORCZAK, 1997, p. 45). Contro ogni possibile pericolo di repressione, anche implicita, nei 
confronti del bisogno di indipendenza dei più piccoli, egli ritenne che l'educazione dovesse partire dai sensi, in primis dalla vista e dal tatto; ogni giorno il neonato imparava a conoscere frammento dopo frammento la madre, partendo dal seno, dal naso, dalle sue mani, per giungere a cogliere progressivamente la sua persona nell'interezza. Le prime conquiste dei piccini erano una sorta di «incantesimi»: i gridolii, la capacità di suzione, l'uso delle mani, comparivano in loro in maniera straordinaria, come fondamentali canali di esplorazione di sé e dell'ambiente in vista di un armonico sviluppo psicologico, cognitivo e morale. L'esplorazione delle proprie mani, oltre ad essere un gesto piacevole svolto in modalità ludica, esprimeva in forma primordiale la nascente volontà di capire e scoprire il mondo con la serietà di uno scienziato impegnato in laboratorio (KORCZAK, 1997, p. 50).

Sulla scia di questo richiamo alla responsabilità educativa, acuito dalle maggiori conoscenze consentite dalla coeva «scoperta del neonato» intrapresa da diverse scienze, Korczak presentò all'interno delle pagine centrali de Il bambino in famiglia la prima bozza di una Magna charta libertatis dei diritti del bambino, poi ripresa nel 1929 nel volumetto Il diritto del bambino al rispetto. Lo fece in un periodo storico carico di presagi negativi sull'infanzia a causa delle conseguenze del primo conflitto mondiale e del sorgere dei primi totalitarismi, ma nel contempo aperto ad una formalizzazione giuridica dei suoi diritti con la Dichiarazione sui diritti dell'infanzia (Ginevra, 1924), benché quest'ultimo atto non fosse risolutivo dei danni provocati dall'adulto come homo rapax. Tre furono, in particolare, i diritti fondamentali individuati da Korczak: 1) «il diritto del bambino alla morte»; 2) «il diritto del bambino alla sua vita presente»; 3) «il diritto del bambino ad essere quel che è» (KORCZAK, 1994).

Quasi parallelamente a Korczak, anche Maria Montessori si rese protagonista di una "rivoluzione pedagogica" avviata con l'esperienza della prima Casa dei bambini, inaugurata nel quartiere S. Lorenzo di Roma il 6 gennaio 1907 (KRAMER, 1988, p. 107-132). Solamente qualche anno più tardi iniziò ad occuparsi di educazione neonatale, ispirandosi al principio della libertà e al riconoscimento della physis del neonato come un essere separato dalla madre e dotato di carattere, intelligenza e sentimento fin dal primo vagito. In un intervento del $1915 \mathrm{ad}$ un congresso a Oakland in California (HONEGGER FRESCO, 2018, p. 30-31), riprendendo alcune osservazioni svolte nelle lezioni raccolte nel volume Antropologia pedagogica (1910), la Montessori sottolineò quanto il contributo dell'igiene infantile fosse di per sé insufficiente nell'interpretare al meglio i bisogni precipui del neonato, in vista di una sua prima educazione secondo natura. Era necessario adottare una prospettiva compiutamente pedagogica, capace di liberare il piccino da ogni possibile condizionamento, per «lasciare fare alla natura il più liberamente possibile» e riconoscere l'infanzia come una «questione sociale» (MONTESSORI, 2016, p. 6-7), secondo quanto declinato nell'opera L'autoeducazione nelle scuole elementari (1916). Grazie ad uno studio continuativo dei neonati nei reparti maternità degli ospedali e delle forme di repressione e di contenimento insite in varie e diffuse pratiche di puericultura, la Montessori pose le basi della sua lotta al disconoscimento dei diritti sociali dell'infanzia. I dati statistici e di demografia storica riferiti al primo ventennio del Novecento in Europa erano dalla sua parte, poiché mostravano una condizione di perdurante miseria materiale e morale di numerose famiglie, la permanenza dei brefotrofi e della pratica del baliatico, un persistente stato di analfabetismo che portava ad ignorare l'esistenza delle più semplici norme di igiene materna e infantile, oltre che l'inefficacia della campagna vaccinale contro la tubercolosi. Lo scoppio della prima guerra mondiale e le conseguenti distruzioni provocarono l'inasprimento di un quadro già di per sé compromesso, reso ancora più drammatico dal successivo sviluppo dei totalitarismi, che intrapresero una lotta alla mortalità e alla morbilità infantile in nome di un'idea di infanzia come «costruzione simbolica e retorica artificiale legata alle politiche di massa del XX secolo, non quindi una categoria biologica né sociologica ma eminentemente politica, come sottolinea il rapporto in cui era posta con la nazione» (GIBELLI, 2005, p. 3). 
La Montessori giunse ad una formulazione organica delle sue idee attorno all'educazione dalla nascita nei primi anni Venti, nell'ambito di alcune conferenze rivolte a famiglie di Bruxelles e Vienna, pubblicate per la prima volta in francese nella rivista «La Femme Belge», e ripubblicate in volume in Austria con il titolo Das Kind in der Familie nel 1923. La pedagogia del neonato che vi delineò, successivamente perfezionata nelle opere L'Enfance (1935) e The absorbent mind (1949), prese le mosse dall'obiettivo di occuparsi della «figura nuova di un bambino incompreso», per mettere in luce gli errori commessi per millenni nei confronti della parte più delicata dell'umanità e prospettare un intervento educativo in grado di tracciare una via nuova. La Montessori focalizzò la sua attenzione soprattutto sul dissidio venutosi a creare, nel corso del tempo, fra l'adulto e il neonato, tale da porre il piccino fin dai primi istanti di vita in uno stato di oppressione, anzi, di repressione - con riferimento non solo al fenomeno psichico studiato dalla psicoanalisi, ma anche agli esiti di quelle forme di «pedagogia nera» esercitate nel corso dei secoli - tanto più pericolosa quanto inconscia, in grado di sopraffare e soffocare le misteriose forze della sua vita interiore (MONTESSORI, 2017, p. 9). I genitori, pronti a sostituirsi a lui in ogni cosa pensando di proteggerlo, non erano in grado di scorgere con un'accurata osservazione come dietro ai suoi gesti, ai suoi sguardi, ai suoi movimenti e alle sue tendenze spontanee si nascondesse un'insopprimibile ricerca di indipendenza. Nulla vi era di più dannoso per il pieno sviluppo della personalità infantile che ignorare questo bisogno psichico di indipendenza, con la conseguenza di costringere il neonato ad una forma di adattamento diretto, forzato e violento al mondo degli adulti, secondo un atteggiamento talmente radicato nella famiglia da sembrare quasi invincibile. Predisporgli fin dal primo respiro un ambiente adatto significava compiere nei suoi confronti un profondo «atto di carità», consapevoli della fragilità comportata dalla «labe originaria» - intesa come peccato originale - che giustificava l'adozione di strategie educative maggiormente rispondenti alle caratteristiche del singolo (MONTESSORI, 2019, p.81).

La questione sollevata dalla Montessori sulla scarsa conoscenza della natura del neonato e, più in generale, del bambino piccolo apriva molteplici implicazioni sul piano pedagogico ma anche sociale, poiché l'incapacità di riconoscere la peculiarità dei bisogni infantili e la permanenza di una visione degli infanti come esseri deboli e privi di una personalità attestavano quanto l'infanzia rappresentasse ancora una «pagina bianca nella storia dell'umanità» (MONTESSORI, 2017, p. 12). Era necessario iniziare, fin dall'ambiente domestico, a porre i pilastri di un' «educazione per la vita», come preparazione del neonato a tutti gli aspetti della vita, nessuno escluso, affinché non incorresse nel pericolo di crescere in un mondo a sé stante, autoreferenziale e, in quanto tale, non pensato per promuovere la sua autonomia (HONEGGER FRESCO, 2018). L'attenzione dedicata dalla Montessori ad una nascita non violenta e alla vita post-natale non era solamente il frutto della sua formazione medica, ma fu finalizzata alla creazione di una vera e propria progettualità pedagogica. Quest'ultima doveva sapersi confrontare con il «dramma» vissuto dal feto nel momento in cui fuoriusciva dal grembo materno, «spossato e ferito come un pellegrino che venga a noi da paesi lontani», ma anche con la sua natura di essere attivo, di «embrione spirituale che si incarna» (MONTESSORI, 2017, p. 25-31), portatore delle direttive del proprio sviluppo futuro secondo determinati periodi sensitivi e profondamente obbediente all'adulto. La sua precoce «intelligenza al lavoro», manifestata simbolicamente nell'uso sempre più raffinato delle mani, rivelava il segreto di cui era portatore ogni piccino, ovvero la libertà.

Tali constatazioni furono successivamente confermate anche dalle osservazioni svolte sui neonati durante il suo soggiorno indiano, durato quasi ininterrottamente dal 1939 al 1949; furono poi raccolte nell'opera The Absorbent Mind, a conferma della natura del neonato come «costruttore dell'uomo», grazie alla presenza di poteri psichici innati e sconosciuti che, se pienamente promossi nei primi tre anni di vita, avrebbero favorito la formazione della personalità umana nella sua interezza (MONTESSORI, 2007, p. 2). Il possesso di una «mente 
assorbente» lo poneva in una condizione di accentuata ricettività, che gli consentiva di costruire in breve tempo la propria «carne mentale», grazie ad un continuo lavorio interiore. Diretto da una potenza misteriosa, per Maria Montessori il neonato «si faceva uomo» per mezzo delle sue mani, cioè per mezzo della sua esperienza nella concretezza del quotidiano.

\section{Conclusioni}

Il tentativo di ricostruire i fili di una storia della pedagogia del neonato in Europa fra l'Umanesimo e il primo Novecento ha consentito di fare i conti, sul piano epistemologico, con la progressiva formalizzazione di un pensiero pedagogico sui primi anni di vita dell'uomo, attraverso lo studio incrociato della genealogia storica e della morfologia personale dei processi di educazione e formazione. Questo processo si è alimentato costantemente ai due forni dell'esperienza educativa e della teoria dell'esperienza educativa, contestualmente all'affermarsi di una nuova concezione dell'infanzia e, conseguentemente, del rapporto fra bambino e adulto, su cui ha pesato la stratificazione plurimillenaria del "sentire", del "pensare" e dell'"agire" umano. Ogni nuovo nato ha iniziato, nel corso del tempo, a non essere più considerato come un infans, cioè un essere mancante, deficitario, un "pappagallo" capace di imitare "goffamente" quanto vede e sente (BECCHI; JULIA, 1996a, p. 30-33), ma un bambino "nuovo" in grado di manifestare nelle diverse circostanze della vita i segni della sua natura autentica di persona umana, benché non abbia ancora acquisito la padronanza nel controllo degli sfinteri, nell'autonomia di deambulazione e di alimentazione, nell'uso della parola. L'attenzione riservata alla costruzione di sé è l'approdo di un percorso che, come si è visto, a partire dalla concezione umanistica di homo artifex trovò nel pensiero di Jean Jacques Rousseau una prima sistematizzazione del principio pedagogico del puerocentrismo, per poi giungere alle soglie del Novecento alla sua declinazione sul piano delle prassi educative, come forma di educazione indiretta e "su misura" dei più piccoli a partire dall'ambiente domestico, grazie al riconoscimento della presenza di una dimensione interiore nell'essere umano fin dalla nascita. In questo modo, un'affermazione come quella di William Wordsworth - «il bambino è padre dell'uomo» - mette in mostra il valore imprescindibile del «lavorio intimo» con cui ogni bambino dà forma alla sua personalità, in quanto protagonista della sua educazione, e non passivo destinatario di un processo di disciplinamento esteriore.

La ricostruzione di alcune tracce di questo percorso, con il richiamo all'opera di autori significativi di ambito europeo nel passaggio dall'Età moderna all'Età contemporanea, consente di rispondere all'esigenza rimarcata a livello storiografico di studiare le bambine e i bambini in qualità di attori della loro stessa storia, tenuto conto delle differenze di genere, religione, ceto sociale, classe economica, cultura della nazione o del gruppo etnico (GECCHELE; POLENGHI; DAL TOSO, 2017, p. 31). Inoltre, può offrire un contributo alla formazione dei genitori e alla professionalizzazione pedagogica degli educatori impegnati nei servizi dell' Early Childhood Education and Care (ECEC), in linea con i nuovi compiti della storia dell'educazione e della pedagogia (POLENGHI; BANDINI, 2016).

\section{Riferimenti bibliografici}

ALBERTI, Leon Battista. I libri della famiglia. Volume II. Torino: Giulio Einaudi Editore, 1994.

ALLEN, Ann Taylor. Spiritual Motherhood: German Feminists and the Kindergarten Movement. 1848-1911. History of Education Quarterly, Cambridge, vol. 22, n. 3, p. 319-339, 1982. 
ANTONIANO, Silvio. Tre libri dell'educatione christiana dei figliuoli. Volume I. In: PATRIZI, Elisabetta. Silvio Antoniano. Un umanista ed educatore nell'età del Rinnovamento cattolico (1540-1603). Volume III. Macerata: EUM 2010.

ARIÈS, Philippe. L'enfant et la vie familiale sous l'Ancien Régime. Paris: Plon, 1960.

BECCHI, Egle; JULIA, Dominique (orgs). Storia dell'infanzia. Volume I. Bari-Roma: Laterza, 1996a.

BECCHI, Egle; JULIA, Dominique (orgs). Storia dell'infanzia. Volume II. Bari-Roma: Laterza, 1996b.

CAGNOLATI, Antonella. L'eredità dell'umanesimo italiano. Pier Paolo Vergerio e le sue teorie educative. Rivista di storia dell'educazione, Firenze, vol. 3, n. 2, p. 93-102, 2016.

CAMBI, Franco; ULIVIERI, Simonetta. Storia dell'infanzia nell'età liberale. Firenze: La Nuova Italia, 1988.

CAMBI, Franco; ULIVIERI, Simonetta (orgs). I silenzi nell'educazione: studi storicopedagogici. Firenze: La Nuova Italia, 1994.

CAROLI, Dorena. Day nurseries \& childcare in Europe, 1800-1938. London: Pelgrave Macmillan, 2017.

COMENIO, Jan Amos. Grande Didattica. Traduzione Anna Biggio. Firenze: La Nuova Italia, 1993.

COVATO, Carmela. La vita privata nella storia dell'educazione. Studi sulla formazione, Firenze, vol. 7, n. 1, p. 73-85, 2004.

CUNNINGHAM, Hugh. Children and Childhood in Western Society since 1500. London: Longman, 1995.

DEMAUSE, Lloyd (org). The History of Childhood. New York: The Psychohistory Press, 1974.

DE MONTAIGNE, Michel. Saggi. Traduzione Virginio Enrico. Volume II. Firenze: Edizioni Casini, 1965.

ERASMO DA ROTTERDAM. L'educazione del principe cristiano. Traduzione Anna Morisi Guerra. Roma: Signorelli, 1992.

ERASMO DA ROTTERDAM. La formazione cristiana dell'uomo. Traduzione Edilia Orlandini Traverso. Milano: Rusconi, 1989.

FÉNELON. L'educazione delle fanciulle. Traduzione Lucia Nutrimento. Treviso: Canova, 1963.

FRIGO, Daniela. Il padre di famiglia. Governo della casa e governo civile nella tradizione dell'«Economica»tra Cinque e Seicento. Roma: Bulzoni, 1985.

FRÖBEL, Friedrich. Mutter-und Koselieder. Dichtung und Bilder zur edlen Pflege des Kinderheitlebens: ein Familienbuch. ed. 3. Berlin: Verlag, 1874. 
GARIN, Eugenio. L'educazione in Europa 1400/1600. Problemi e programmi. 3. ed. RomaBari: Laterza, 1976.

GARIN, Eugenio. L'educazione umanistica in Italia. Roma-Bari: Laterza, 1964.

GECCHELE, Mario; POLENGHI, Simonetta; DAL TOSO, Paola (orgs). Il Novecento: il secolo del bambino? Parma: Junior-Spaggiari, 2017.

GIBELLI, Antonio. Il popolo bambino: infanzia e nazione dalla grande guerra a Salò. Torino: Einaudi, 2005.

GOODY, Jack. The development of the Family and Marriage in Europe. Cambridge: Cambridge University Press, 1983.

HAAS, Louis. The Renaissance Man and His Children. Childbirth and Early Childhood in Florence 1300-1600. Basingstoke: Macmillan, 1998.

HEROARD Jean. Journal de Jean Héroard, sous la direction de Madeleine Foisil. Volume 1. Paris: Fayard, 1989.

HILL, Christopher. Society and Puritanism in Pre-Revolutionary England. London: Secker and Warburg, 1964.

JAEGER, Werner. Paideia. La formazione dell'uomo greco. Traduzione Luigi Emery e Alessandro Setti. 2. ed. Milano: Bompiani, 2003.

KORCZAK, Janusz. Il diritto del bambino al rispetto. Traduzione Giovanni Frova. Milano: Luni Editrice, 1994.

KORCZAK, Janusz. Come amare il bambino. I. Il bambino in famiglia. Traduzione Margherita Bacigalupo. Milano: Luni Editrice, 2018.

KRAMER, Rita. Maria Montessori. A biography. Da Capo Press: New York, 1988.

LASCARIDES, V. Celia; HINITZ, Blythe F. History of Early Childhood Education. ed. 3. London-New York: Taylor \& Francis Ltd., 2011.

LOCKE, John. Pensieri sull'educazione. Traduzione Mario Barbagallo. Milano: Corbaccio, 1937.

LUTERO, Martin. Opere scelte, 4. Scuola e cultura. Compito delle autorità doveri dei genitori (1524 e 1530). Traduzione Maria Cristina Laurenzi. Torino: Claudiana, 1990.

MONTESSORI, Maria. L'autoeducazione nelle scuole elementari. Milano: Garzanti, 2016.

MONTESSORI, Maria. Il peccato originale. Brescia: Scholé-Morcelliana, 2019.

MONTESSORI, Maria. Il bambino in famiglia. Milano: Garzanti, 2017.

MONTESSORI, Maria. La mente del bambino. Mente assorbente. Milano: Garzanti, 2007. 
NECKER DE SAUSSURE, Albertine. Educazione progressiva. Traduzione Elena Ederle. Brescia: La Scuola, 1940.

PANCERA, Carlo. Il pensiero educativo di Fénelon. Firenze: La Nuova Italia, 1991.

PESTALOZZI, Johann Heinrich. Sull'infanticidio. Traduzione Giulia Di Bello. Firenze: La Nuova Italia, 1999.

PESTALOZZI, Johann Heinrich. Buch der Mütter oder Anleitung für Mütter ihre Kinder bemerken und reden zu lehren. Erstes heft. Zurich und Bern: Gessner, 1803.

PESTALOZZI, Johann Heinrich. Madre e figlio. L'educazione dei bambini. Traduzione Giovanni Sanna. Venezia: La Nuova Italia, 1927.

PIRONI, Tiziana. Da Ellen Key a Maria Montessori: la progettazione di nuovi spazi educativi per l'infanzia. Ricerche di pedagogia e didattica, Bologna, vol. 5, n. 1, p. 1-15, 2010

POLENGHI, Simonetta. Ruoli parentali e sentimento dell'infanzia in età moderna. La Famiglia, Brescia, vol. 36, n. 206, p. 1-20, 2001.

POLENGHI, Simonetta; BANDINI, Gianfranco. The History of education in its own lights: signs of crisis, potential for growth. La storia dell'educazione di fronte a se stessa: segnali di crisi, direzioni di crescita. Espacio, Tiempo y Educación, Salamanca, vol. 3, n. 1, p. 3-20, 2016. DOI: https://doi.org/10.14516/ete.2016.003.001.2

PRÜFER, Johannes. Federico Fröbel. Traduzione Gian Pietro Sanna. Venezia: La Nuova Italia, 1927.

ROUSSEAU, Jean Jacques. Emilio, o dell'educazione. Traduzione Andrea Potestio. Roma: Studium, 2016.

RUTSCHKY, Katharina. Pedagogia nera. Fonti storiche dell'educazione civile. Traduzione Paolo Perticari. Milano: Mimesis, 2015.

SANI, Roberto. For a History of Family Education in the Modern and Contemporary Era. Research Itineraries and Perspectives. History of Education \& Children's Literature, Macerata, v. 1, n. 1, p. 55-81, 2006.

SCAGLIA, Evelina. La scoperta della prima infanzia. Per una storia della pedagogia 0-3. Volume II. Roma: Studium, 2020.

SHORTER, Edward. The making of the Modern Family. New York: Basic Books, 1975.

STONE, Lawrence. The Family, Sex and Marriage in England 1500-1800. New York: Harper \& Row, 1977.

VIVES, Juan Luis. The education of a Christian woman: a sixteenth-century manual. Traduzione Charles Fantazzi. Volume I. Chicago: University of Chicago Press, 2000. 